\title{
Multidisciplinary approach to maximize angiogenesis and wound healing using piezoelectric surgery, concentrated growth factors and photobiomodulation for dental implant placement surgery involving lateral wall sinus lift: two case reports.
}

Nghiem V. Doan, 1, 2, a, @ Thang Q. Huynh, 3, b, @ Sang Tran, 3 Gui Wang, 2 Stephen Hamlet, 1 Van Dau, 4 Dzung Dao, 4 Nam-Trung Nguyen, 5 Hong T. Nguyen, 6 Joshua Doan, 2 Thanh Thai, 3 Nha Doan, 3 Khue N. Truong, 3

@ corresponding author, \& equal contributor

Vascular Cell. 2020; 12(1):2 | C Nghiem V. Doan, Thang Quyet Huynh, Sang Tran, Gui Wang, Stephen Hamlet, Van Dau, Dzung Dao, Nam-Trung Nguyen, Hong T. Nguyen, Joshua Doan, Thanh Thai, Nha Doan, Khue N Truong

Received: 06 September 2019 | Accepted: 02 February 2020 | Published: 11 June 2020

Vascular Cell ISSN: 2045-824X

DOI: https://doi.org/10.24238/13221-12-1-186

\section{Author information}

1. School of Dentistry and Oral Health - Griffith University; Mount Gravatt, QLD 4122, Australia

2. Department of Mechanical Engineering - St Lucia, QLD 4072, Australia

3. Can Tho University of Medicine and Pharmacy; Can Tho, Vietnam

4. School of Engineering and Built Environment - Griffith University; Mount Gravatt, QLD 4122, Australia

5. Queensland Micro and Nanotechnology Centre - Griffith University; Mount Gravatt, QLD 4122, Australia

6. Hong Bang International University; Ho Chi Minh, Vietnam

[a] nghiem.doan@grffiith.edu.au

[b] thanghuynhphd@yahoo.com 


\section{Abstract}

Background : The combined application of surgical and therapeutic modalities to improve the rate and or degree of healing and tissue regeneration is gaining in popularity.

Aim : This study describes two case reports using piezoelectric surgery (PES), concentrated growth factors (CGF) and photobiomodulation (PBM) as a means of enhancing angiogenesis and tissue regeneration following dental implant sinus lift procedures.

Materials and Methods: Sinus lateral windows were created using PES and the sinus membrane raised to make a new partition. Dental implants were inserted concurrently and packed with autologous fibrin rich CGF. Wound sites underwent PBM treatment using a multiwave locked system laser. The efficacy of the multidisciplinary approach was assessed by radiographic and clinical evaluation one, three, six, ten, and twelve-months post-surgery.

Results: Postoperative revival was ordinary with no noteworthy postoperative complications. Wound closure and angiogenesis occurred shortly after day one. New bone formation in all augmented maxillary sinuses was detected alongside the implants on plain radiographs and on conebeam computed tomograms. The patients were highly satisfied $(>80 \%)$ with the treatment regime and the success rate of the implants was $100 \%$ after a usual 10 months loading period.

Conclusion : PBM, PES, and CGF promoted angiogenesis and wound healing following lateral wall sinus lift by increasing wound approximation and decreasing bleeding and swelling. Patients recorded less postoperative pain, speech impairment, analgesic use and trismus.

\section{Keywords}

photobiomodulation - piezoelectric surgery - concentrated growth factors - sinus augmentation

- angiogenesis - wound healing

\section{Introduction}

Posterior maxillary tooth loss leads to pneumatisation of the sinus which diminishes the vertical component of bone. Maxillary lateral window sinus lift is a procedure commonly used to augment the hard tissue volume prior to implant placement in the maxillary posterior region. The traditional approach for maxillary sinus lift involves surgical entry across the lateral wall of the maxilla using rotary instrumentation, followed by raising the sinus membrane and incorporation of bone graft under direct visualization. However, the use of rotary instruments often results in a high incidence of sinus membrane perforation. On the other hand, piezoelectric instruments which can perform similar functions have fewer such complications [1].

To assist in the subsequent healing process fibrinrich gels which are derived from extracts of blood derived concentrated growth factors (CGF), have been utilized to hasten new bone formation in guided bone regeneration and sinus graft procedures [2]. The use of CGF in dentistry although a relatively new concept, has been successfully used to enhance regeneration and healing for a

\section{Materials and methods}

\section{Patient selection}

Patients were included if they met the following inclusion criteria: 1) The patient planned for variety of procedures such implant placement, socket preservation, bony reconstruction, and tissue regeneration [3, 4]. This suggests combining piezoelectric surgery (PES) with CGF may synergistically enhance both wound healing and angiogenesis.

A further innovative approach to improve wound healing combines PES and CGF treatment with photobiomodulation (PBM). PBM promotes extra cellular energy generation and self-repair through the use of red or near infrared light and is designed to enhance angiogenesis, healing progression and tissue regeneration, while also reducing inflammation and pain. The successful clinical application of PBM has been well documented in numerous reports [5]. However, no previous reports have documented the effects of a combined multidisciplinary approach using PES and CGF with PBM as a method of enriching angiogenesis in tissue regeneration following a dental implant sinus lift procedure. This report documents the findings of two cases using this novel approach.

maxillary sinus lateral lift procedure for implant placement; 2) The inserted implants were to be restored with fixed prostheses; 3) Favorable and balanced dental occlusal pattern, 4) A minimal post extraction wound healing of three months; 5) Easy access to carry a sinus lift surgical procedure; 6 ) 
Ideal sinus anatomy such as missing intra-sinus septa or arteries in the planned treatment; 7) No evidence of infection in sinus or Schneiderian membrane.

The study participants consisted of two male patients aged 53 and 57 years. In both cases, evidence of severe alveolar bone resorption was found in the posterior maxillary areas required for implant placement (Figures $1 \&$ 2). The diagnostic plan comprised an implant risk and periodontal assessment; diagnostic casts for surgical and prosthetic planning; cone beam computed tomography (CBCT) for anatomical survey and bone quantity evaluation.

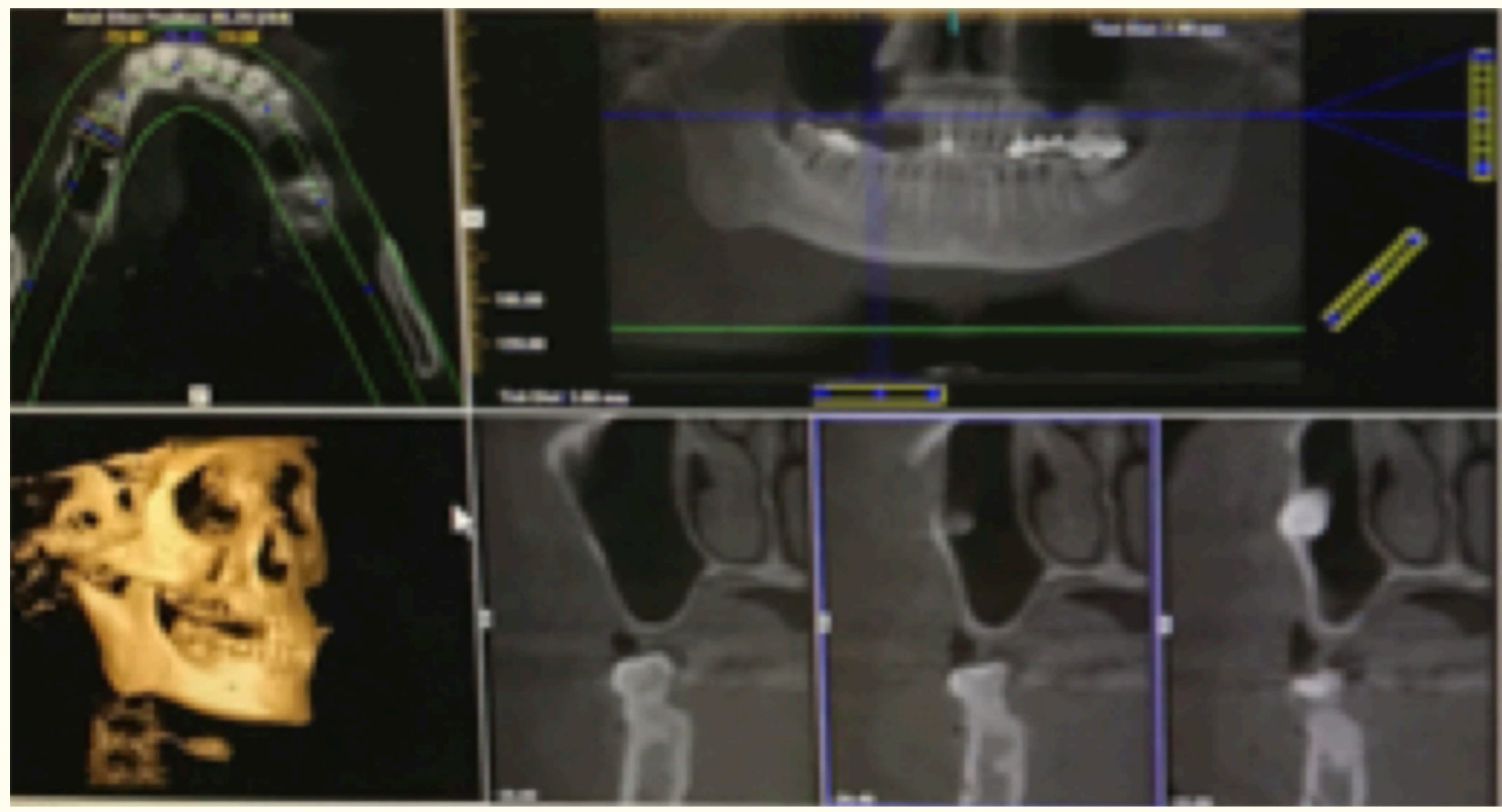

Figure 1

\section{Figure 1}

Case 1 (left - right, top - bottom). Cone Beam Computed Tomography (CBCT) views of the skull at different cuts (Axial view, OPG, 3D reconstruction, and coronal views show missing teeth at upper right quadrant resulting in severe right maxillary sinus pneumatisation and alveolar bone atrophy. 

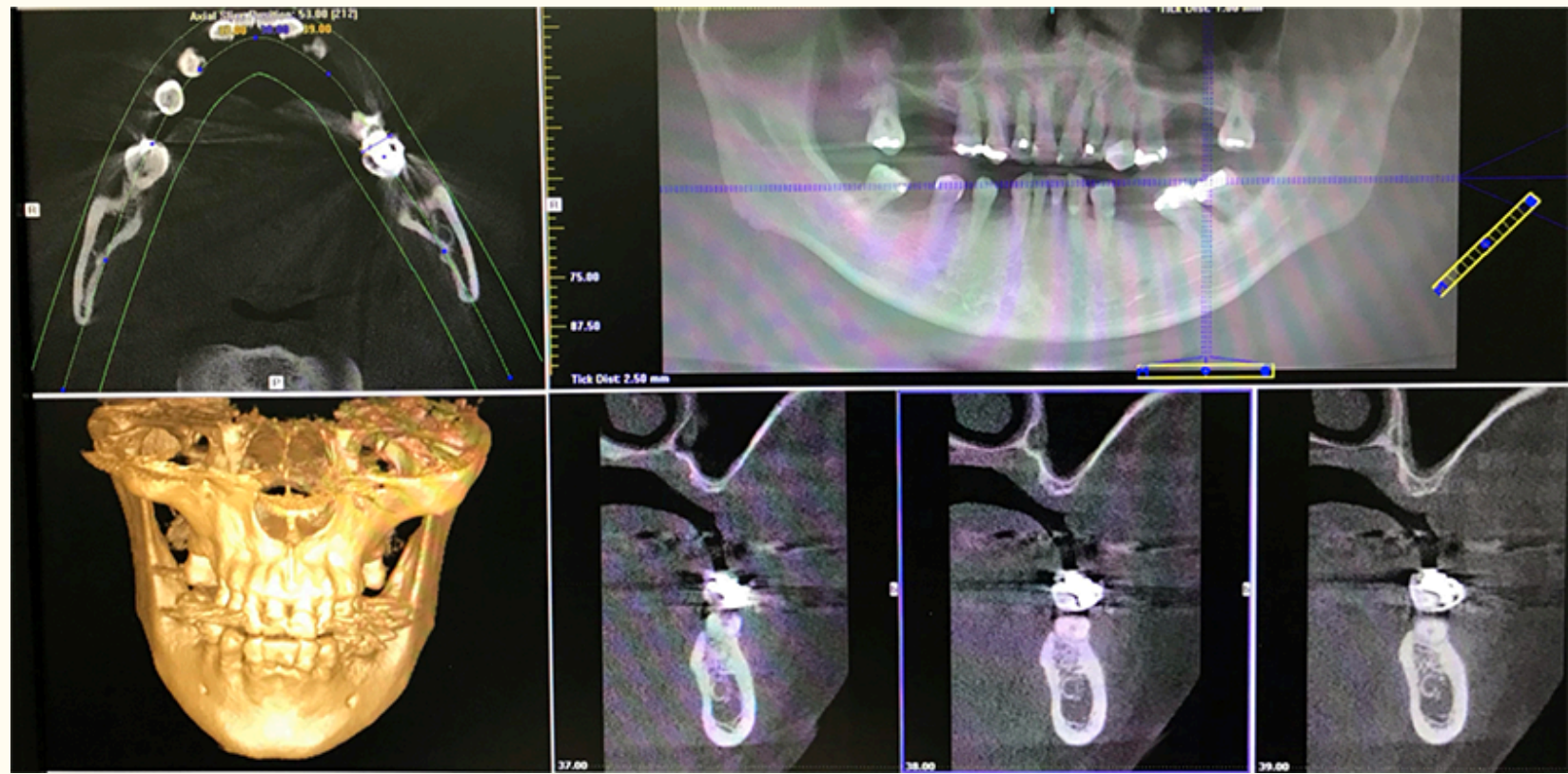

Figure 2

\section{Figure 2}

Case 2 (left - right, top - bottom). Cone Beam Computed Tomography (CBCT) views of the skull at different cuts (Axial view, OPG, 3D reconstruction, and coronal views) . CBCT showed advanced sinus pneumatization and severe alveolar bony atrophy at the upper canine, first and second premolars areas.

\section{Concentrated growth factors (CGF)}

Prior to surgery, $40 \mathrm{~mL}$ of venous blood was collected and aliquoted into two red and two white top sterile $10 \mathrm{~mL}$ Vacutainer ${ }^{\circledR}$ tubes. These four tubes were immediately centrifuged in a Medifuge MF 200 (Silfradent, Italy) following the manufacturer's instructions. Following centrifugation, the vacutainers were left for 20 minutes before additional processing. The final CGF mixture was acquired by removing the top platelet poor plasma and meticulously collecting the clot layer (Figure 3 ). Using a sterile Petri dish, the CGF clot was detached from any red blood portion using scissors, which could then be available for subsequent applications []]. Fabrication of CGF 'sticky bone' and membrane were followed per the manufacturer's protocol. For sticky bone, bovine demineralized freeze-dried bone (BioOss ${ }^{\circledR}$, Chatswood, Australia) and hydroxyapatite and calcium triphosphate (Genoss ${ }^{\circledR}$, Seoul, Republic of Korea,) were used instead of calcium triphosphate. 


\title{
Concentrated growth factors (CGF)
} 4 -phase separation

\author{
Serum
}

Buffy coat
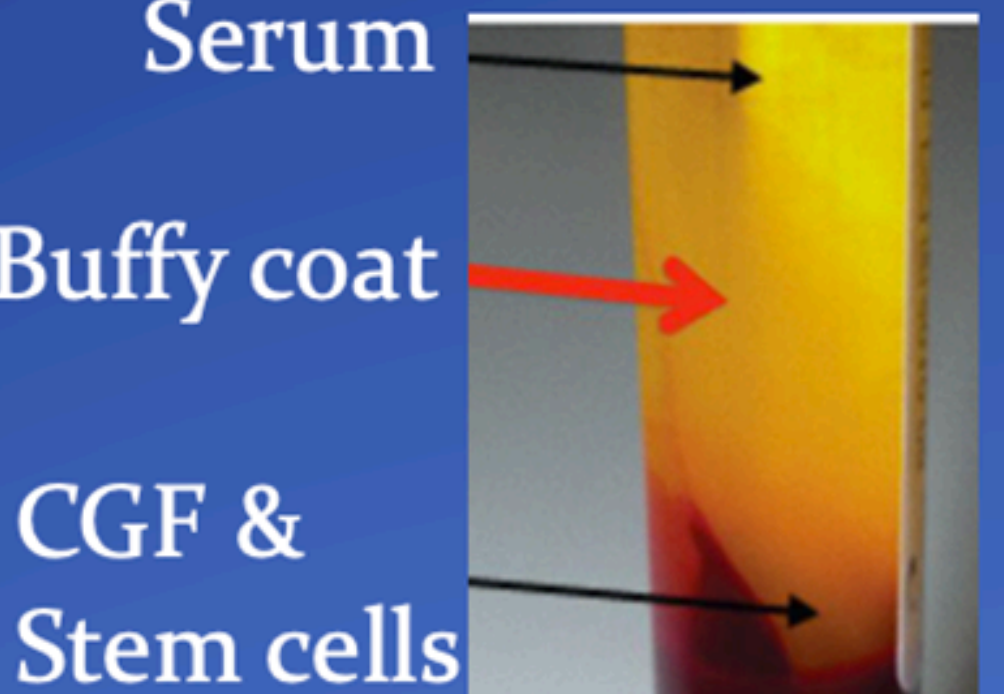
incision was made extending from the distal surface of canine to the mesial surface of the upper wisdom teeth. A vertical incision was protracted until the end of the buccal vestibule. To ensure safe access to the lateral sinus, a full-thickness mucoperiosteal flap beginning from the mid-crestal region or somewhat toward the palatal edge was selected. A broad base releasing incision at the front or posterior point of this flap was devised to warrant a proper blood supply from the base (Figures $4 \& 5$ ).

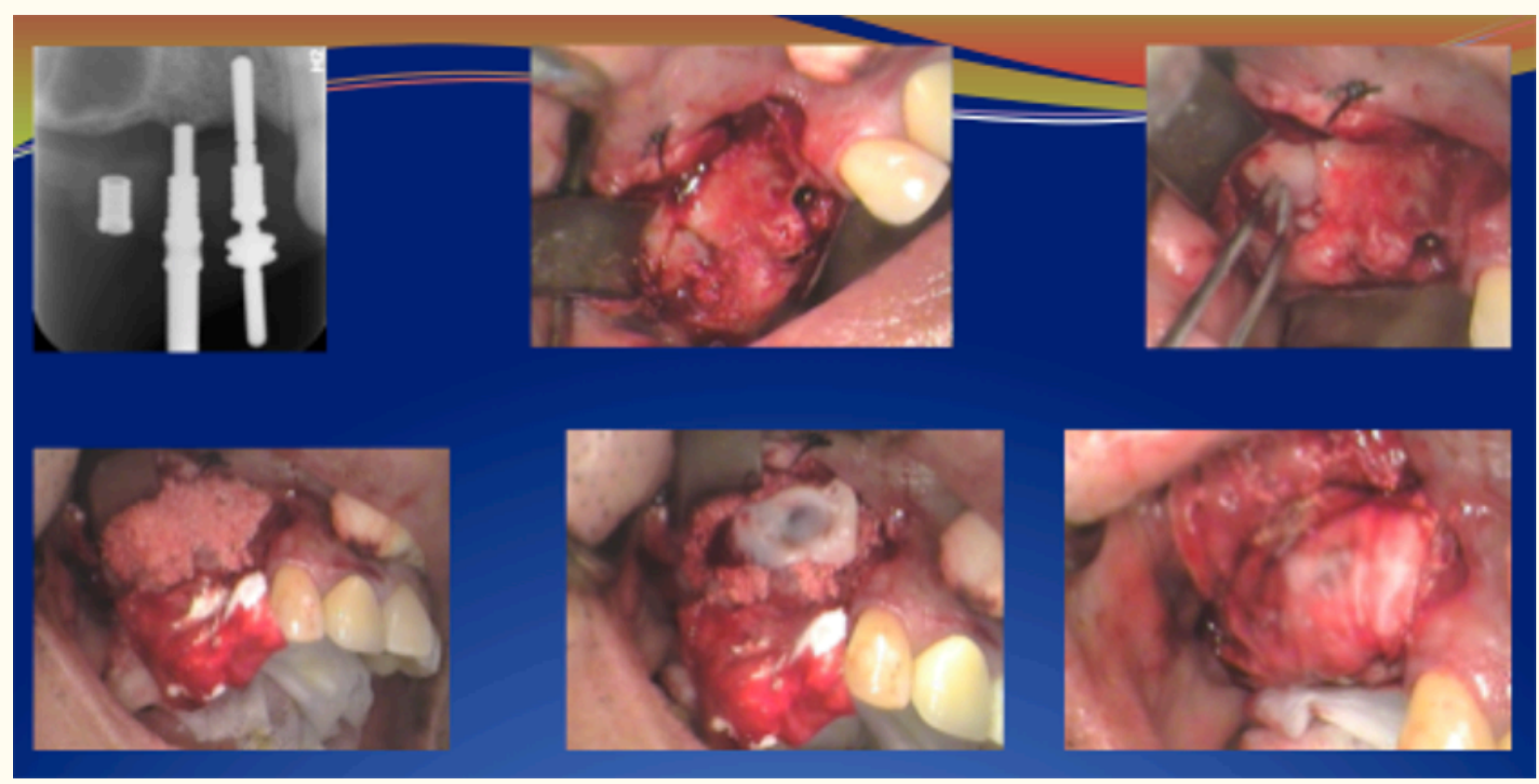

Figure 4

\section{Figure 4}

Case 1. Outline of treatment (clockwise top to bottom) for three dental implants (\#13, \#14, \#15) using piezoelectric cut lateral sinus window lift procedure in conjunction with CGF sticky bone and fibrin membrane. 


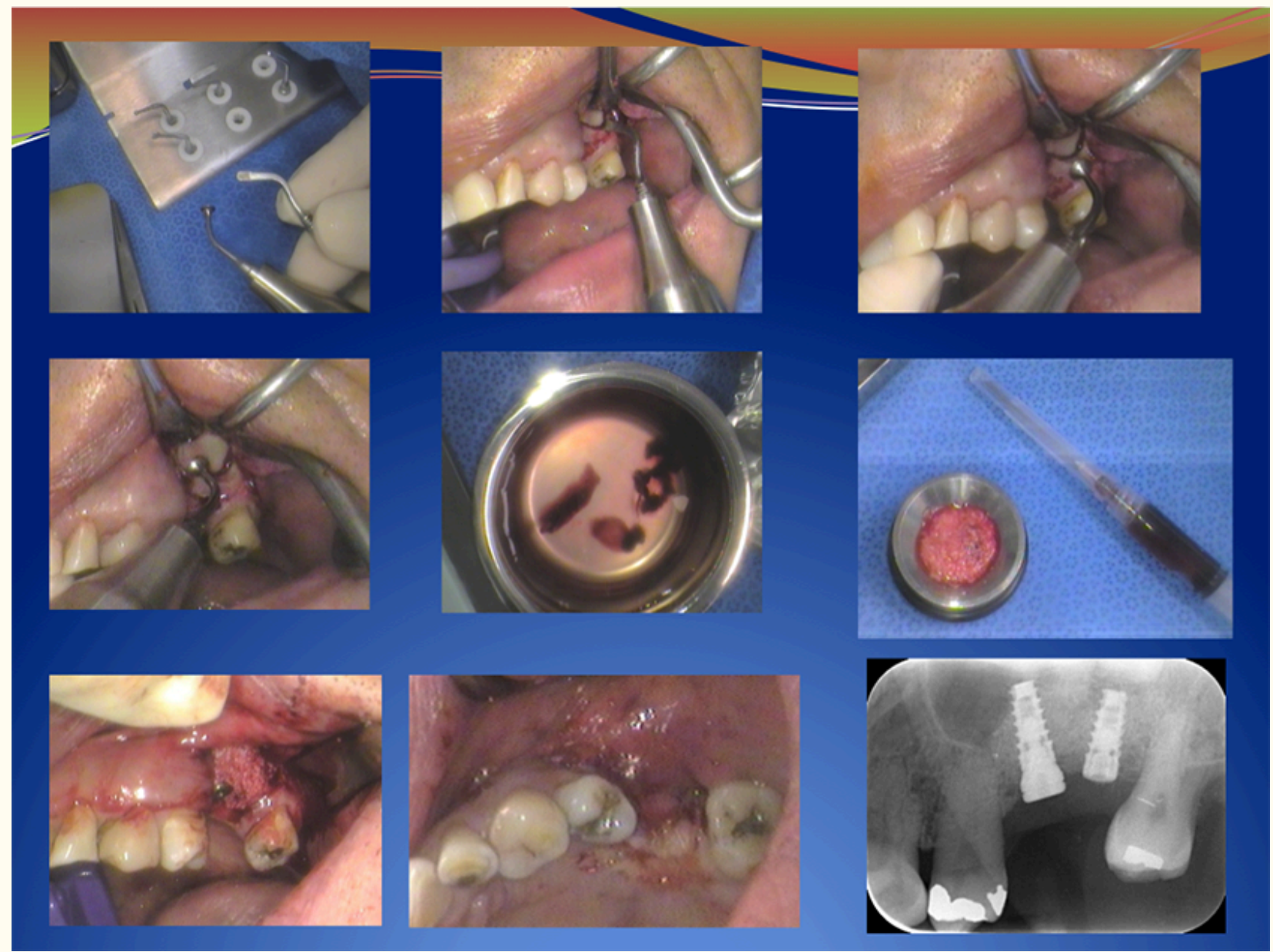

Figure 5

\section{Figure 5}

Case 2. Outline of Treatment - For upper left first and second molar using CGF sticky bone and membrane.

The surgical approach for sinus lifting required the planning of a trap door from the lateral sinus wall to lift the Schneiderian membrane. Under complete disclosure of the lateral maxillary wall, an antrostomy was created in the lateral sinus wall to obtain entry to the sinus membrane. To achieve an exposed the trap-door window, the piezoelectric method was used to deliver sufficient access to eradicate the slender to dense cortical bone and to uncover the delicate sinus membrane, thus permitting an area to be formed for housing the bone graft material. After the entire bone trap window was created, the Schneider membrane lift using a Piezosurgery tip and the final membrane lift were done manually by the utilization of suitable elevators. Upon completion of membrane elevation, its integrity was validated using a nasal pinch test.
Subsequently, a CGF fibrin membrane was used to shield the roof of the newly formed sinus cavity (Figures $4 \& \underline{5}$ ).

Also, this lift needed to spread anteriorly-posteriorly to deliver the uncovered sinus floor to allow for graft and implant placement. Dental implants were then placed in specified locations (Figure 6 ) and the area produced under the elevated sinus membrane grafted with various fillers comprising of CGF, autogenous bone, bone substitute or a combination of these materials. The wound areas were then covered with a CGF mixture of fibrin rich sticky bone and membrane (Biogide collagen membrane, Geistlich Pharma, Switzerland) prior to final closure with slow resorbable sutures (MonoSyn, B. Braun Surgical S.A., Spain). 


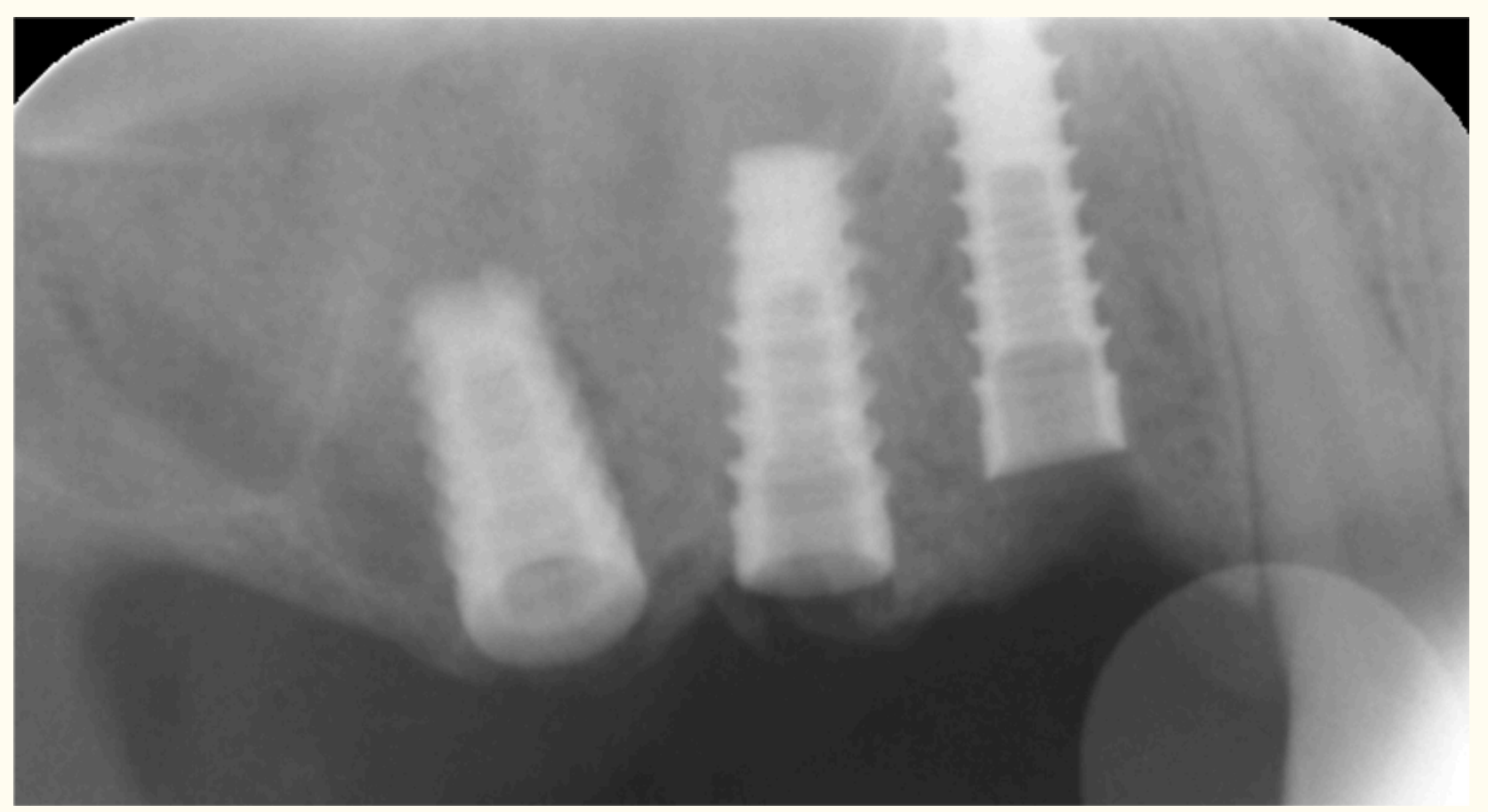

Figure 6

\section{Figure 6}

Implant placement. Post-surgical peri-apical digital radiography revealed sinus deficiencies (case

1) were packed with CGF containing materials.

\section{Photobiomodulation}

The surgical sites were irradiated with an MLS laser (ASA laser, Vicenza, Italy) per the manufacturer's protocol. The entire CPW energy used was 0.637 Joules at an energy density of $1.27 \mathrm{~J} / \mathrm{cm} 2$, frequency of $1500 \mathrm{~Hz}$, and a lasing time per application of 0.04 seconds at $25 \%$ capacity. Multiple laser applications were employed at the apical, buccal, coronal, lingual, mesial, and distal aspects of the surgical wound.

\section{Assessment of healing}

The patients were provided with detailed postsurgery oral hygiene and dietary instructions. Digital radiographic and clinical assessments were performed at the one, three, six, ten, and twelvemonth reviews. During these, the patients were

\section{Results}

Post-surgical recovery was ordinary with no significant postoperative complications. No sinus perforation, infection or allergies were noted, and wound closure and angiogenesis occurred shortly after day one. This was evident from the increased blood flow seen in the Infrared Vein finder images asked to report levels of postoperative pain, swelling, bleeding, speech impairment, analgesic use, trismus, and wound healing, as well as their overall satisfaction at one day, one week, and four weeks post-surgery. Visual Analogue Scale (VAS) was used to measure post-surgical pain, with 1 being the least and 10 being the worst. Overall satisfaction was employed to monitor post-surgical satisfaction, with 0 being the least and 100 being the most.

To measure surgical wound blood flow and vascularity, an infra-red vein finder (Guangzhou Meca Co, China) was used. To quantify implant integration and stability, this study determined removal torque values (MIS Implant, Shlomi, Israel), periotest values (Medizintechnik Gulden, Modautal/ Germany) and radio frequency analysis (Penguin RFA, Aseptico, WA USA).

of the surgical wound after one week in both cases. Table 1 summarizes the changes in clinical indices over one-year post-operation and patient reported information for the two reported cases.

New bone formation in all augmented maxillary sinuses was detected alongside the implants using plain radiographs and cone-beam computed 
tomograms. There was also significant improvement in the following clinical indices 6 months following surgery compared with those at the time of implant placement: Removal Torque value $(<35 \mathrm{Ncm}$ vs $>35 \mathrm{Ncm})$, Perio Test Value $(+1$ to +9 vs -8 to 0 ), and ISQ readings ( $<63$ vs $>75$ ). Both patients were very satisfied with the overall treatment $(>80 \%)$, and the success rate of implant was $100 \%$ after a usual 10 -month loading period.

When asked to compare their experiences with the current technique, patients indicated that PBM, PES, and CGF helped wound healing by speeding up wound approximation. There was a perception of a reduction in post-surgical complications such as postoperative pain ( 3 days instead of 6 days) and swelling ( 3 days instead of 5 days), with less bleeding, minimal speech impairment and less analgesic use. Little trismus and fast angiogenesis/ wound healing were also reported. Over the following six months, a uniform and stable building of freshly produced bone was noted through digital imaging.

\section{Table 1}

Summary of clinical indices from two case reports of the application of piezoelectric surgery, concentrated growth factors and photobiomodulation for sinus lift and dental implant placement: $(+/-)$ improved / worse; Visual Analogue Scale ( 0 - 10); Periotest [-8 (least mobile) to +20 (most mobile)]; RFA [<63(less stable) to $>75$ (more stable)].

\begin{tabular}{|c|c|c|c|c|c|c|c|c|c|c|c|c|c|c|c|c|}
\hline & & & \multicolumn{12}{|c|}{ Assessment schedule } & \multirow[t]{2}{*}{ Overall } \\
\hline & & \multicolumn{2}{|c|}{ Day 0} & \multicolumn{2}{|c|}{ Day 1} & \multicolumn{2}{|c|}{ Day 7} & \multicolumn{2}{|c|}{$\begin{array}{l}\text { Day } 30 \\
\text { (1month) }\end{array}$} & \multicolumn{2}{|c|}{$\begin{array}{l}\text { Day 90 } \\
\text { (3mths) }\end{array}$} & \multicolumn{2}{|c|}{$\begin{array}{l}\text { Day } 180 \\
\text { (6mths) }\end{array}$} & \multicolumn{2}{|c|}{$\begin{array}{l}\text { Day } 365 \\
\text { (12mths) }\end{array}$} & \\
\hline \multicolumn{2}{|c|}{\begin{tabular}{|l} 
Case number \\
\end{tabular}} & $\mathrm{C} 1$ & $\mathrm{C} 2$ & $\mathrm{C} 1$ & $\mathrm{C} 2$ & $\mathrm{C} 1$ & $\mathrm{C} 2$ & $\mathrm{Cl}$ & $\mathrm{C} 2$ & $\mathrm{Cl}$ & $\mathrm{C} 2$ & $\mathrm{C} 1$ & $\mathrm{C} 2$ & $\mathrm{C} 1$ & $\mathrm{C} 2$ & \\
\hline \multicolumn{2}{|c|}{ Post-surgical recovery } & + & + & $2+$ & $2+$ & $3+$ & $3+$ & $4+$ & $4+$ & $5+$ & $5+$ & $6+$ & $6+$ & $6+$ & $6+$ & Excellent \\
\hline \multicolumn{2}{|c|}{ Complications } & No & No & No & No & No & No & No & No & No & No & No & No & No & No & None \\
\hline \multicolumn{2}{|c|}{ Closure / angiogenesis } & + & + & $2+$ & $2+$ & $3+$ & $3+$ & $4+$ & $4+$ & $5+$ & $5+$ & $6+$ & $6+$ & $6+$ & $6+$ & Excellent \\
\hline \multirow{6}{*}{$\begin{array}{l}\text { Visual } \\
\text { Analogue } \\
\text { Scale }\end{array}$} & Pain & 8 & 8 & 8 & 7 & 0 & 0 & 0 & 0 & 0 & 0 & 0 & 0 & 0 & 0 & Excellent \\
\hline & Swelling & 8 & 8 & 9 & 9 & 2 & 2 & 0 & 0 & 0 & 0 & 0 & 0 & 0 & 0 & Minimal \\
\hline & Bleeding & 5 & 4 & 4 & 3 & 2 & 2 & 0 & 0 & 0 & 0 & 0 & 0 & 0 & 0 & Minimal \\
\hline & \begin{tabular}{|l}
$\begin{array}{l}\text { Speech } \\
\text { impairment }\end{array}$ \\
\end{tabular} & 7 & 6 & 6 & 5 & 2 & 2 & 0 & 0 & 0 & 0 & 0 & 0 & 0 & 0 & Minimal \\
\hline & $\begin{array}{l}\text { Analgesic } \\
\text { use }\end{array}$ & 8 & 7 & 8 & 6 & 0 & 0 & 0 & 0 & 0 & 0 & 0 & 0 & 0 & 0 & Less \\
\hline & Trismus & 7 & 5 & 7 & 5 & 0 & 0 & 0 & 0 & 0 & 0 & 0 & 0 & 0 & 0 & Minimal \\
\hline \multicolumn{2}{|c|}{ \% Satisfaction } & 87 & 90 & 90 & 95 & 92 & 98 & 100 & 100 & 100 & 100 & 100 & 100 & 100 & 100 & $\begin{array}{l}\text { Very } \\
\text { good }\end{array}$ \\
\hline \multicolumn{2}{|c|}{$\begin{array}{l}\text { Bone formation } \\
\text { (digital x-rays) }\end{array}$} & $6+$ & $6+$ & & & & & $4+$ & $4+$ & $4+$ & $4+$ & $4+$ & $4+$ & $5+$ & $5+$ & Excellent \\
\hline \multicolumn{2}{|c|}{$\begin{array}{l}\text { Bone formation } \\
\text { (CBCT) }\end{array}$} & $6+$ & $6+$ & & & & & & & & & $5+$ & $5+$ & & & Excellent \\
\hline \multirow{2}{*}{\multicolumn{2}{|c|}{ Removal torque value }} & $<35$ & $<35$ & & & & & & & $>35$ & $>35$ & & & & & Good \\
\hline & & 4 & 3 & & & & & & & & & 6 & 5 & & & Good \\
\hline \multicolumn{2}{|c|}{$\begin{array}{l}\text { Radio Frequency } \\
\text { Analysis } \\
\text { (RFA) }\end{array}$} & $<65$ & $<65$ & & & & & & & & & $>65$ & $>65$ & & & Good \\
\hline \multicolumn{2}{|c|}{ Number of implants } & 3 & 2 & & & & & & & & & 3 & 2 & 3 & 2 & Same \\
\hline
\end{tabular}

\section{Discussion}

The results from these two case reports, despite their statistical power limitation, are a useful reflection of this innovative approach to enhance angiogenesis, wound healing and minimize postoperative complications. The absence of sinus perforation or post-operative infection also demonstrates the 'gentle impact' of piezoelectric surgery in the trap-door lateral sinus approach for implant treatment similar to that reported in previous studies [3, 4]. Furthermore, the rapid wound closure and angiogenesis, as indicated by increased blood flow to the surgical wound after one week in both cases, suggests the combined multidiscipline approach of using PBM, PES, and CGF has significant potential for tissue regeneration in dental implant sinus lift surgery.

New bone formation in the augmented maxillary 
sinuses alongside the implants, as well as the increase in implant stability from the time of placement to that six months later, echoed the success of the above-mentioned method in line with previous studies [7]. This was further substantiated by the $100 \%$ success rate of the implants after 10 months loading. The experience and aptitude of the operating surgeon was also one of the main factors contributing to the success of these two cases as evident from the high overall patient satisfaction scores. This again is in agreement with the results from a previously published case report [8].

The mechanism of action of the combined PES, CGF and PBM treatment was not considered as it outside the scope of this study. However, animal research findings have shown that CGF can be a crucial factor in angiogenesis, as fibrin accrued in hypodermal tissue encourages angiogenesis [9]. In the process of wound healing and wound closure, angiogenic capillary sprouts grow into the fibrin/ fibronectin-rich wound clot in a span of few days where they establish into a microvascular network through the granulation tissue [10].

Numerous methods of clot preservation have been recognized including guided bone regeneration and guided tissue regeneration utilizing autografts, allografts, and xenografts. Recently, the novel use of CGF has been well acknowledged [11, 4]. Growth factors, such as proteins, are innately appearing constituents that are capable of stimulating cellular development, proliferation, healing, differentiation, and angiogenesis [12]

Blood growth factors chiefly originate in blood plasma and platelets. Among these, the essential growth factors are platelet derived growth factor (PDGF), transforming growth factor (TGF), vascular endothelial growth factor (VEGF), epidermal growth factor (EGF), and insulin-like growth factor 1 (IGF 1) $[13,14]$. It has been documented that blood obtained through patient venipuncture can be used to generate fibrin-rich gel (PRF) $[4,6,15,16]$. CGF clots have been shown to have excellent tensile strength, additional growth factors, superior viscosity, and better adhesive strength than PRF [17]. The application of autologous fibrin offers several benefits including being a safe, easy, and inexpensive procedure without side effects $[17,18]$.

Multiwave-Locked System (MLS) lasers such as the Mphi laser used in this study, mixes waves both continuous (808 $\mathrm{nm}$ for enriched anti-inflammatory and anti-edema effects) and pulsed (905 $\mathrm{nm}$ boosts analgesic properties). This makes it one of the most efficient lasers for enlightening wound healing,

\section{Conclusion}

A multi-discipline approach using PES, CGF and PBM has been shown in this report of two cases to be an innovative method to enrich angiogenesis and wound healing in tissue regeneration. It may also present potential solutions to dilemmas such as discomfort, morbidity, implant failure, compatibility, especially in post-operative CGF regenerative oral surgical techniques [4]. The MLS laser currently has several therapeutic indications including sprains, tendinitis, muscle tears, craniofacial pain, brachial neuralgia, bursitis, lumbago, arthritis, articular pain, edema, and hematoma.

The two reported cases are a novel attempt at reconstructing and restoring maxillary bony atrophy in combination with the application of synthetic alloplastic bone substitutes, ancillary surgical techniques, and biocompatible additives. The available data encountered in this topic proposed that recently formed bone of acceptable quality (density) and quantity is produced within three to six months with minimal postoperative complications [8]. Besides, the technique is economically suitable to the dental patients with lateral window sinus lift as the cost of using MLS laser and producing CGF products are insignificant compare to the potential hidden cost of correcting sinus surgery complications.

Normally, regeneration of bony defects in the atrophic posterior maxilla is, at times, linked to complications such as sinus perforation, shrinkage of blood clot, serum exudation, development of 'lifeless gaps', and the risk of minor infection. These have considerably affected the regenerative progression of the maxillary bone [4]. The outcomes of this case report shed light on ways to overcome shortfalls in sinus lift procedures. CGFs are applicable alone or when mixed with bone graft material. Application of piezoelectric, CGF sticky bone and membrane is therefore one of the most current methods for reconstruction of bone defects in the maxillary sinus area [4, 8, 19]. Additional use of PBM further enhances the prospect of clinical success. The results of the two case reports have demonstrated their efficiency in shortening bonehealing time in large bone defects, reducing the incidence of postoperative complications and enabling better restoration of nearby periodontium. The method is relatively simple with minimal risk of infection and allergic reaction and is economically feasible.

Through the combined application of PMB, PES, and CGF, this study had taken advantage of their clinical properties as a means of enhancing angiogenesis and wound healing in tissue regeneration in a dental implant lateral wall sinus lift procedure. Ideally, a further randomized split mouth clinical trial to compare the effectiveness of PES, CGF and PBM treatment is required to test these observations on tissue regeneration in the atrophic maxilla.

and cost found in traditional methods. Further research, particularly a prospective split mouth study and randomized clinical trials need to occur to confirm its effectiveness and efficacy in tissue regeneration in dental implant sinus lift procedures. 


\section{Copyright permissions}

\section{Original submitted files for images}

Below are the links to the authors' original submitted files for images.

\section{Original image file for Figure 1}

Click here to view.

Original image file for Figure 2

Click here to view.
The image copyright permissions are solely the responsibility of the authors.

Original image file for Figure 3

Click here to view.

Original image file for Figure 4

Click here to view.

Original image file for Figure 5

Click here to view.

Original image file for Figure 6

Click here to view.

\section{References}

1. Jordi C, Mukaddam K, Lambrecht JT, Kühl S. Membrane perforation rate in lateral maxillary sinus floor augmentation using conventional rotating instruments and piezoelectric device-a meta-analysis. International Journal of Implant Dentistry. 2018;4:1-9.

2. Gandhi Y. Sinus Grafts: Science and TechniquesThen and Now. Journal of maxillofacial and oral surgery. 2017;16:135-144.

3. Kao S, Lui M, Cheng D, Chen T. Lateral trap-door window approach with maxillary sinus membrane 1 lifting for dental implant placement in atrophied edentulous alveolar ridge. Journal of the Chinese Medical Association. 2014;78:85-88.

4. Doan N, Nguyen-Pham L, Liang C, Duong QT. A review on the application of concentrated growth factors and MPhi laser to regenerate oral defects in the oral and maxillofacial region and a two cases report. International Journal of Oral \& Maxillofacial Surgery. 2017;46:203-204.

5. Hosseinpour S, Fekrazad R, Arany PR, Ye Q. Molecular impacts of photobiomodulation on bone regeneration: A systematic review. Progress in biophysics and molecular biology. Progress in biophysics and molecular biology. 2019.

6. Choukroun J, Adda F, Schoeffler C, Vervelle A. Une opportunite en paro-implantologie: le PRF. Implantodontie. 2000;42:55-62.

7. Troedhan A, Schlichting I, Kurrek A, Wainwright $M$. Primary implant stability in augmented sinus liftsites after completed bone regeneration: $\mathrm{A}$ randomized controlled clinical study comparing four subantrally inserted biomaterials. Scientific Reports. 2014;4:5887-.

8. Barfield Luca Barbera L, Mat E, Ahmed M. Sinus Lift Procedure and Immediate Implant Placing: A Piezo-Surgery and Platelet Rich Plasma Approach: A Case Report. Otolaryngol (Sunnyvale). 2017;7(6):335-.
9. Baranski J Engineering Patterns to Study Vascular Biology PhD Thesis University of the Pennsylvania Scholarly Commons 2012 Baranski J. Engineering Patterns to Study Vascular Biology - PhD Thesis University of the Pennsylvania Scholarly Commons 2012.

10. Tonnesen MG, Feng $X$, Clark RAF. Angiogenesis in Wound Healing. Journal of Investigative Dermatology Symposium Proceedings. 2000;5:40-46.

Mansour P, Kim P. Use of Concentrated Growth Factor (CGF) in implantology. Australasian Dental Practice. 2010;21(2):162-176.

12. Clark RA. Fibrin and wound healing. Ann N Y Acad Sci. 2001;936:355-67.

13. Lazić Z, Bubalo M, Petković-Curcin A, Dukat M, Mihajlović B. Therapeutic use of platelet-rich plasma in oral surgery. Vojnosanit Pregl. 2009;66(10):821-5.

14. Plachokova AS, Nikolidakis D, Mulder J, Jansen JA, Creugers NH. Effect of platelet-rich plasma on bone regeneration in dentistry: a systematic review. Clin Oral Implants Res. 2008;19(6):539-45.

15. Choukroun J, Diss A, Simonpieri A, Girard M, Schoeffler C, Dohan SL, et al. Platelet-rich fibrin (PRF): a second-generation platelet concentrate. Part IV: clinical effects on tissue healing. Oral Surg Oral Med Oral Pathol Oral Radiol

Endod. 2006;101(3):56-60.

16. Choukroun J, Diss A, Simonpieri A, Girard M, Schoeffler C, Dohan SL, et al. Platelet-rich fibrin (PRF): a second-generation platelet concentrate. Part V: histologic evaluations of PRF effects on bone allograft maturation in sinus lift. Oral Surg Oral Med Oral Pathol Oral Radiol Endod. 2006;101(3):299-303.

17. Sohn DS, Moon JW, Moon YS, Park JS, Jung HS. The use of concentrated growth (CGF) for sinus augmentation. J Oral Implant. 2009;38:25-38. 
18. Mirković S, Đurđević-Mirković T, Petrović L, Božić $D$. The use of concetrate growth factors in gyded bone regeneration after lateral sinus lift procedure (case report). HealthMED. 2013;7(2):700-4.
19. Mirković S, Djurdjević-Mirković T, Puškar T. Application of concentrated growth factors in reconstruction of bone defects after removal of large jaw cysts - The two cases report. Vojnosanit Pregl. 2015;72(4):368-371.

\section{Copyright \& License}

Statement: Copyright (c) 2020, Nghiem V. Doan, Thang Quyet Huynh, Sang Tran, Gui Wang, Stephen Hamlet, Van Dau, Dzung Dao, Nam-Trung Nguyen, Hong T. Nguyen, Joshua Doan, Thanh Thai, Nha Doan, Khue N Truong.

Holder: Nghiem V. Doan, Thang Quyet Huynh, Sang Tran, Gui Wang, Stephen Hamlet, Van Dau, Dzung Dao, Nam-Trung Nguyen, Hong T. Nguyen, Joshua Doan, Thanh Thai, Nha Doan, Khue N Truong

Licensee: Publiverse Online S.R.L.

License: Open Access This article is distributed under the terms of the Creative Commons AttributionNonCommercial 4.0 International License (https://creativecommons.org/licenses/by-nc/4.0/), which permits unrestricted use, distribution, and reproduction in any medium for non-commercial purposes, provided you give appropriate credit to the original author(s) and the source, provide a link to the Creative Commons license, and indicate if changes were made. The Creative Commons Public Domain Dedication waiver (https://creativecommons.org/publicdomain/zero/1.0/) applies to the data made available in this article, unless otherwise stated.

\section{Vascularcell P7PUBLIVERSE}

The present article has been published in Vascular Cell journal by Publiverse Online S.R.L. 